\title{
A robust AFM-based method for locally measuring the elasticity of samples
}

\author{
Alexandre Bubendorf ${ }^{*}$, Stefan Walheim ${ }^{2,3}$, Thomas Schimmel ${ }^{2}$ and Ernst Meyer ${ }^{1}$
}

\section{Full Research Paper}

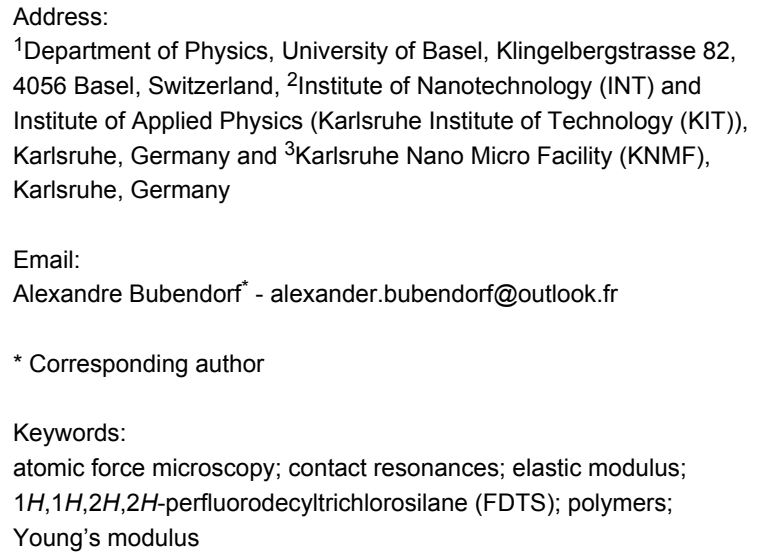

\author{
Beilstein J. Nanotechnol. 2018, 9, 1-10. \\ doi:10.3762/bjnano.9.1 \\ Received: 21 June 2017 \\ Accepted: 06 December 2017 \\ Published: 02 January 2018 \\ Associate Editor: U. D. Schwarz \\ (C) 2018 Bubendorf et al.; licensee Beilstein-Institut. \\ License and terms: see end of document.
}

\begin{abstract}
Investigation of the local sample elasticity is of high importance in many scientific domains. In 2014, Herruzo et al. published a new method based on frequency-modulation atomic force microscopy to locally determine the elasticity of samples (Nat. Commun. 2014, 5, 3126). This method gives evidence for the linearity of the relation between the frequency shift of the cantilever first flexural mode $\Delta f_{1}$ and the square of the frequency shift of the second flexural mode $\Delta f_{2}{ }^{2}$. In the present work, we showed that a similar linear relation exists when measuring in contact mode with a certain load $F_{N}$ and propose a new method for determining the elastic modulus of samples from this relation. The measurements were performed in non-dry air at ambient temperature on three different polymers (polystyrene, polypropylene and linear low-density polyethylene) and a self-assembled monolayer of $1 H, 1 H, 2 H, 2 H-$ perfluorodecyltrichlorosilane (FDTS) on a silicon oxide substrate perforated with circular holes prepared by polymer blend lithography. For all samples the relation was evidenced by recording $\Delta f_{1}, \Delta f_{2}$ and $F_{N}$ as a function of the $Z$-displacement curves of the piezoelectric scanner. The occurence of a plastic deformation followed by an elastic deformation is shown and explained. The necessary load $F_{N}$ for measuring in the elastic domain was assessed for each sample, used for mapping the frequency shifts $\Delta f_{1}$ and $\Delta f_{2}$ and for determining the elastic modulus from $\Delta f_{2}{ }^{2} / \Delta f_{1}$. The method was used to give an estimate of the Young's modulus of the FDTS thin film.
\end{abstract}

\section{Introduction}

Knowledge of the local elasticity of samples is of high interest in many scientific domains, as many processes and physical quantities are correlated with the elastic modulus. In biology, for instance, studies showed that the elasticity of cells depends on their age, the stage of the cell cycle and the degree of differentiation [1]. In physics, the band gap size of nanocrystals and 
the presence of planar defects on nanotubes are a function of the Young's modulus [2,3]. Probing local elasticity requires an instrumentation capable of operating with high resolution and under different conditions, such as variable temperature, pressure or humidity. Since its invention, the atomic force microscope (AFM) [4] has confirmed its value for locally determining nanomechanical properties, such as the Young's modulus, of the sample surface. Initially, the measures were done qualitatively, with the cantilever operated in intermittent-contact mode by showing the phase-shift contrast between regions of different elasticities [5]. This was followed by quantitative measurements using various static and dynamic methods [6,7]. Although the results obtained with these methods are in good agreement with theoretical data and data obtained from macroscopical experiments, difficulties in the precise determination of the elastic modulus based on the theoretical model, or during the use of the method may be encountered when using dynamicmode AFM. This is the case with the methods devised by Hurley and Turner [6] and Herruzo and co-workers [7]. In Hurley and Turner's [6] method, the stated equations for the computation of the normal sample stiffness by numerical methods (analytical expression for normal sample stiffness formulated by Bubendorf [8] and given in Supporting Information File 1) used to determine sample elasticity are based on the equations established by Rabe [9] and Rabe et al. [10] for atomic force acoustic microscopy (AFAM) [11-14]. They describe the dynamics of a clamped cantilever elastically coupled with the sample surface at its tip end. These equations have the disadvantage of strongly depending on the dimensions of an ideal beam-shaped cantilever. However, most cantilevers used for measurements are not ideal. Thus, to achieve consistent results, the values of length and tip height have to be corrected. When measurements using the multifrequency AFM $[15,16]$ method of Herruzo et al., which is based on the excitation of two cantilever eigenmodes [17-21], are performed in non-dry air, the instability of the tip-sample distance feedback loop, due to the use of the frequency shift as control parameter, makes the application of the method difficult if not impossible. However, despite these disadvantages, both methods are particularly interesting because of the complementarity of their advantages. The method of Hurley and Turner [6], which is based on tracking the first flexural and torsional contact resonances, has the advantage of staying stable even if the measurements are performed in non-dry air. In contrast, the method of Herruzo et al. [7] uses a simple theoretical model that depends only weakly on the dimensions of the cantilever. We present a new dynamic method for measuring the sample elasticity [8] that combines the simplicity of the theoretical model of Herruzo et al. [7] with the robustness of the measuring method based on contact resonances. Since the range of elasticity values of polymers is covered by the domain of validity of the theoretical model, those values were used for testing the new method.

\section{Principle of the Method Theoretical model}

The method is based on the theoretical model developed by Herruzo et al. [7] for the computation of the effective elastic modulus of samples $E_{\text {eff }}$ ranging from $1 \mathrm{MPa}$ to $3 \mathrm{GPa}$ from the measured frequency shifts of the two flexural modes of a cantilever operated in intermittent-contact mode:

$$
E_{\mathrm{eff}}=\sqrt{\frac{8}{R A_{1}}} \frac{k_{2}^{2} f_{0,1}}{k_{1} f_{0,2}^{2}} \frac{\Delta f_{2}^{2}\left(d_{m}\right)}{\Delta f_{1}\left(d_{m}\right)},
$$

where $R$ is the effective tip radius and $k_{i}, A_{i}, f_{0, i}$ and $\Delta f_{i}\left(d_{m}\right)$ are, respectively, the force constant, the oscillation amplitude, the resonance frequency and the frequency shift of the $i$-th flexural mode in free space as a function of $d_{m}$, the closest distance between tip and sample in an oscillation cycle.

The validity of the relation and the stability of the microscope during its operation is ensured by the use of an amplitude $A_{2}$ small enough compared to $A_{1}$ (ideally, $A_{1}$ is at least one order of magnitude larger than $A_{2}$ ). The applied normal force on the sample is controlled by controlling $\Delta f_{1}$, so that $\Delta f_{2}$ changes as a function of sample elasticity. In our method based on tracking the contact resonances, the applied normal force $F_{N}$ is directly controlled by controlling the vertical deflection of the cantilever. We can hence rewrite the previous equation as

$$
E_{\text {eff }}=\alpha\left(A_{1}\right) \frac{\Delta f_{2}^{2}\left(F_{N}\right)}{\Delta f_{1}\left(F_{N}\right)}
$$

where $\alpha$ is a parameter that depends on amplitude $A_{1}$, determined from a sample of known elastic modulus. $\Delta f_{1}$ and $\Delta f_{2}$ are the frequency shifts that depend on the applied normal force, $F_{N}$. They are determined from the measured contact resonances through the relation $\Delta f_{i}=f_{i}-f_{0, i}$, where $f_{i}$ is the contact resonance of the $i$-th flexural mode.

Herruzo et al. [7] showed that if the Young's modulus of the tip $E_{\text {tip }}$ is at least two orders of magnitude larger than that of the sample $E_{\text {sample }}$ then

$$
E_{\text {sample }} \approx E_{\text {eff }} .
$$

\section{Feedback controls}

As introduced by Herruzo et al. [7], five different feedback loops are used as feedback controls, as shown in Figure 1: two 
feedback loops for keeping the amplitudes $A_{1}$ and $A_{2}$ constant, two feedback loops for keeping the phase shifts $\phi_{1}$ and $\phi_{2}$ constant in order to track the contact resonances $f_{1}$ and $f_{2}$, and the last feedback loop as main feedback for controlling the applied normal force $F_{N}$.

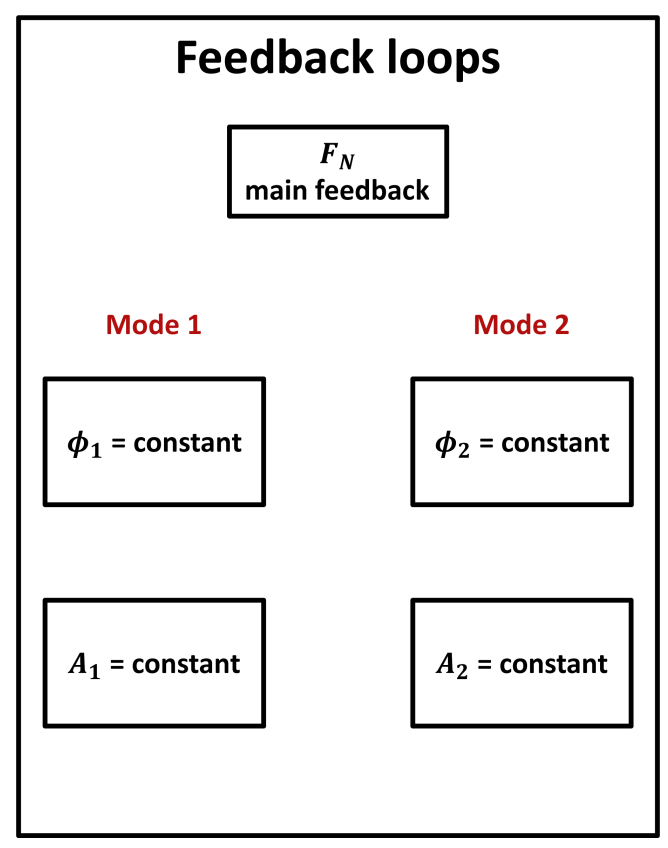

Figure 1: Feedback loops necessary to track the flexural contact resonances of the cantilever. The main feedback loop ensures that the measurements are performed with a constant normal force. The other feedback loops are used to maintain constant values of phase $\phi_{1}$ and amplitude $A_{1}$ of the first flexural mode, and of phase $\phi_{2}$ and amplitude $A_{2}$ of the second flexural mode.

\section{Experimental}

\section{Microscope and data acquisition}

The measurements were performed with a flex AFM head from Nanosurf and the Nanonis scanning probe microscope controller. The system integrates the phase-locked loops (PLLs) necessary for tracking the contact resonances and all the controlling and signal generation modules for measuring and mapping the physical quantities. Square areas of $2.5 \mu \mathrm{m} \times 2.5 \mu \mathrm{m}$ were scanned at a resolution of $256 \times 256$ pixels and a scan speed of $2 \mu \mathrm{m} \cdot \mathrm{s}^{-1}$.

\section{Cantilevers}

The measurements were performed with a PPP-CONT cantilever from Nanosensors $^{\mathrm{TM}}$ (Si tip of Young's modulus $\left.E_{\text {tip }} \approx 169 \mathrm{GPa}\right)$ characterized by $f_{0,1}=16.35 \mathrm{kHz}$, $k_{1}=0.324 \mathrm{~N} \cdot \mathrm{m}^{-1}, Q_{1}=68, f_{0,2}=102.98 \mathrm{kHz}, k_{2}=13.1 \mathrm{~N} \cdot \mathrm{m}^{-1}$, $Q_{2}=208$, where $Q_{i}$ is the $Q$ factor of the $i$-th flexural mode in free space. The force constants were computed for $k_{1}$ from the theoretical formula $k_{1}=\left(E w t^{3}\right) /\left(4 l^{3}\right)$ [22], where $E, t, w$ and $l$ are, respectively, Young's modulus, thickness, width and length of the cantilever beam, and for $k_{2}$ by using the relation $k_{2}=40.4 k_{1}$ established by Rast and co-workers [23]. The thickness $t=2.35 \mu \mathrm{m}$ was determined from $f_{0,1}$ and the formula $t \approx 6.1911(\rho / E)^{(1 / 2)} f_{0,1} l^{2}$ [22]. Width $w=52 \mu \mathrm{m}$ and length $l=445 \mu \mathrm{m}$ of the cantilever were determined by optical microscopy. The values $E=169 \mathrm{GPa}$ and $\rho=2,330 \mathrm{~kg} \cdot \mathrm{m}^{-3}$ were taken for the Young's modulus of the silicon beam and its mass density. The measured normal force $F_{N \text {,meas }}$, which corresponds to the vertical deflection of the cantilever and gives the value of the applied normal force $F_{N}$ on the sample surface was calibrated with a factor obtained by dividing the cantilever spring constant by the cantilever optical sensitivity $S_{z}$. The optical sensitivity of value $229 \mathrm{~nm} \cdot V^{-1}$ was determined by measuring a vertical deflection-distance curve on an n-type silicon(111) sample of electrical resistivity $=10 \Omega \cdot \mathrm{m}$ and by taking the inverse of the slope. The two first flexural modes of the cantilever in contact were excited with the amplitudes $A_{1}=22 \mathrm{mV}$ and $A_{2}=5 \mathrm{mV}$ for all samples.

\section{Samples and measurement conditions}

Three different polymers (polystyrene (PS), polypropylene (PP) and linear low-density polyethylene (LLDPE)) and a selfassembled monolayer (SAM) of $1 H, 1 H, 2 H, 2 H$-perfluorodecyltrichlorosilane (FDTS) on a silicon oxide $\left(\mathrm{SiO}_{x}\right)$ substrate were investigated. The SAM was prepared with circular holes obtained by polymer blend lithography (PBL) [24]. A reference sample consisting of polytetrafluoroethylene (PTFE), commonly called Teflon, with a nominal Young's modulus of $500 \mathrm{MPa}$ was used to determine parameter $\alpha\left(A_{1}\right)$. The measurements were performed in non-dry air with a relative humidity of $36 \%$ at an ambient temperature of $27^{\circ} \mathrm{C}$.

\section{Results and Discussion Force-distance and frequency shift-distance curves}

Before starting the investigation of the sample elasticity, a force-distance curve and a frequency shift-distance curve (Figure 2 and Figure 3, left) for both flexural modes were recorded for each sample to determine for which values of $F_{N}$ the relation between $\Delta f_{1}$ and $\Delta f_{2}^{2}$ is linear and to compute the parameter $\alpha\left(A_{1}\right)$. The measured applied normal force $F_{N \text {,meas }}$ and frequency shifts $\Delta f_{1}, \Delta f_{2}$ were recorded for a displacement of the piezoelectric scanner in the normal $Z$-direction from 0 to $350 \mathrm{~nm}$. To measure the curves, the cantilever tip first indented the sample to a depth corresponding to a displacement of the $Z$-scanner of $350 \mathrm{~nm}$ and both PLLs were then switched on. The curves were recorded during retraction of the tip in order to avoid unlocking the PLLs. This would happen if the tip indented the sample from a starting position out of contact, as contact resonances are quite far from those in free space. 


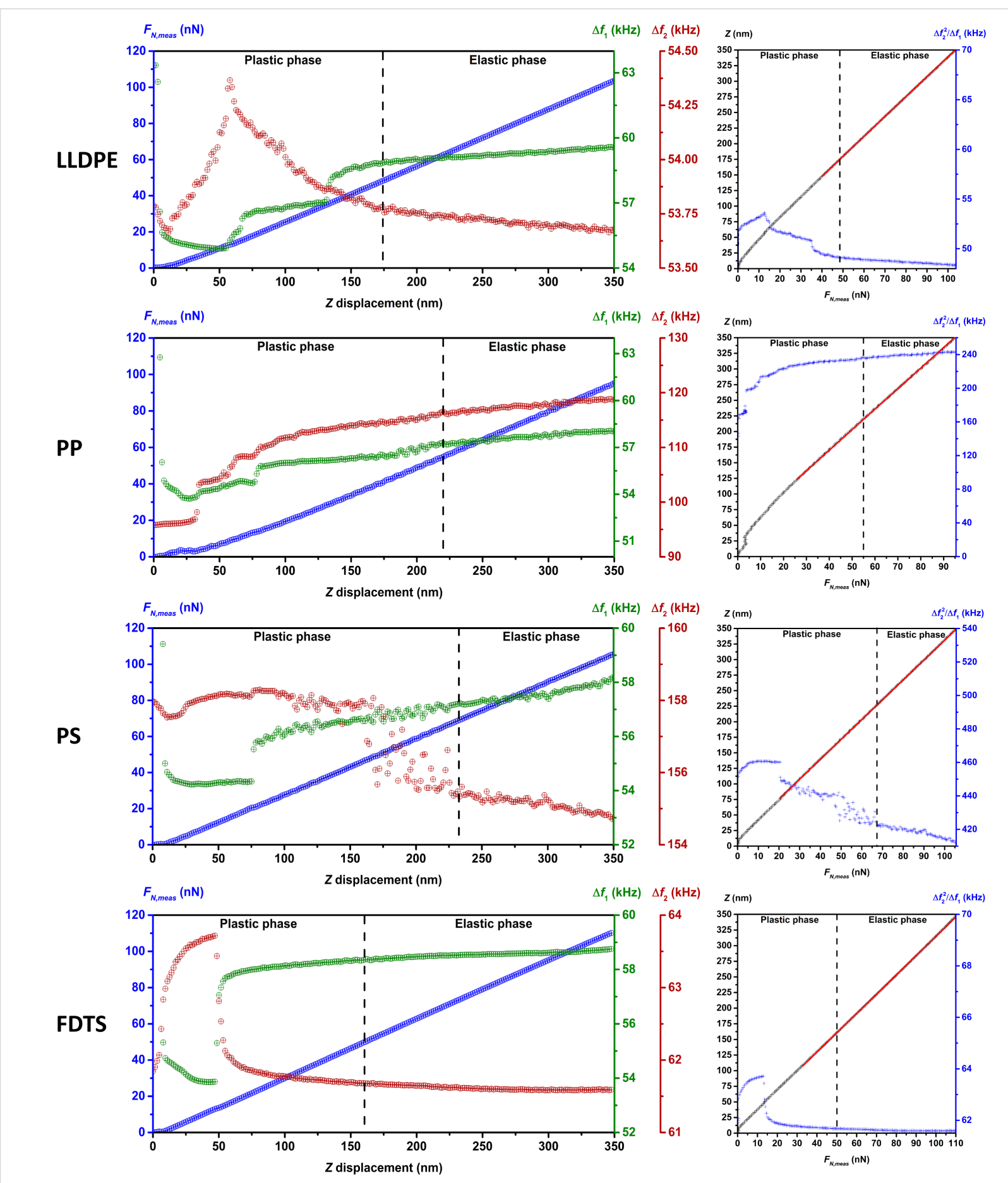

Figure 2: Left: Force-distance and frequency shift-distance curves of LLDPE, PP, PS and FDTS for the determination of the minimal normal force $F_{N}$ used as setpoint for the elasticity measurements. The curves for $\Delta f_{1}$ and $\Delta f_{2}$ as functions of the Z-displacement evidence the existence of a plastic deformation phase followed by an elastic deformation phase. Right: The fitting of the $Z\left(F_{N \text {,meas }}\right)$ curves gave a value close to the cantilever spring constant showing the excessive compliance of the cantilever. The $\Delta f_{2}^{2} / \Delta f_{1}\left(F_{N \text {,meas }}\right)$ curves show quasi-invariance of the ratio $\Delta f_{2}^{2} / \Delta f_{1}$ in the elastic deformation phase and in the highest region of the plastic deformation phase.

In the frequency shift-displacement curves, we observe first a nonlinear relation between the measured frequency shifts, $\Delta f_{1}$ and $\Delta f_{2}$, and the displacement of the scanner, followed by a linear relation. This suggests a plastic deformation phase of the sample surface during the first step of the indentation, followed by an elastic deformation phase. Analysis of the force-displacement curve evidences the same relations with, however, a linear relation in the nonlinear domain of the frequency shift-displace- 

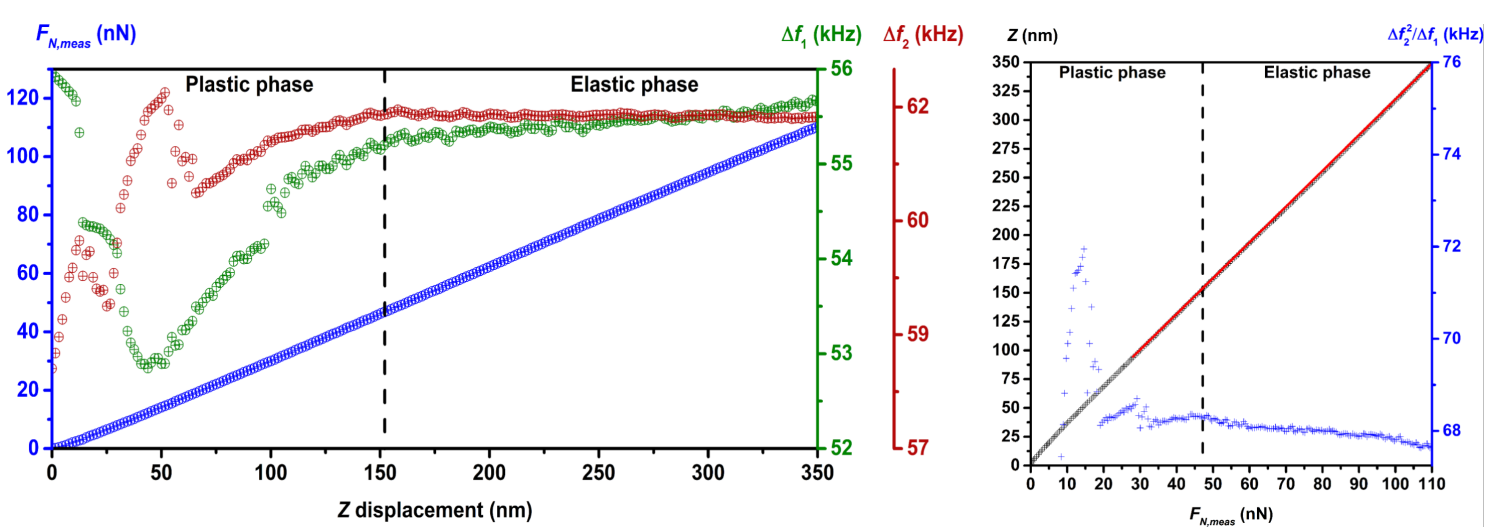

Figure 3: Left: Force-distance and frequency shift-distance curves of PTFE. The curves of $\Delta f_{1}$ and $\Delta f_{2}$ as a function of the $Z$-displacement evidence the existence of a plastic deformation phase followed by an elastic deformation phase. Right: The fitting of the $Z\left(F_{N \text {,meas }}\right)$ curve gave a value close to the cantilever spring constant showing the excessive compliance of the cantilever. The $\Delta f_{2}^{2} / \Delta f_{1}\left(F_{N \text {,meas }}\right)$ curves show quasi-invariance of the ratio $\Delta f_{2}^{2} / \Delta f_{1}$ in the elastic deformation phase and in the highest region of the plastic deformation phase. The parameter $\alpha\left(A_{1}\right)$ determined by dividing the nominal value of the elasticity of the reference sample by the mean value of the ratio in the elastic deformation phase gave a value of $7,355 \mathrm{~Pa} \cdot \mathrm{Hz}{ }^{-1}$.

ment curves. This is explained by the low spring constant of the cantilever in comparison to the normal sample stiffness, beginning at a certain $Z$-displacement value.

A good model of the cantilever in contact with the sample surface is two springs in series, $k_{1}$ and $k_{\text {sample,norm, representing }}$ the spring constant of the cantilever and the normal sample stiffness (of constant value in the elastic phase), respectively (Figure 4a). As shown in Figure 4b, the applied normal force generated by the $Z$-displacement $\Delta z$, is $F_{N}=k_{\text {eff }} \Delta z$, where $k_{\text {eff }}$ is the effective spring of value $k_{1} k_{\text {sample,norm }} /\left(k_{1}+k_{\text {sample,norm }}\right)$. As the normal stiffness of sample increases during indentation,
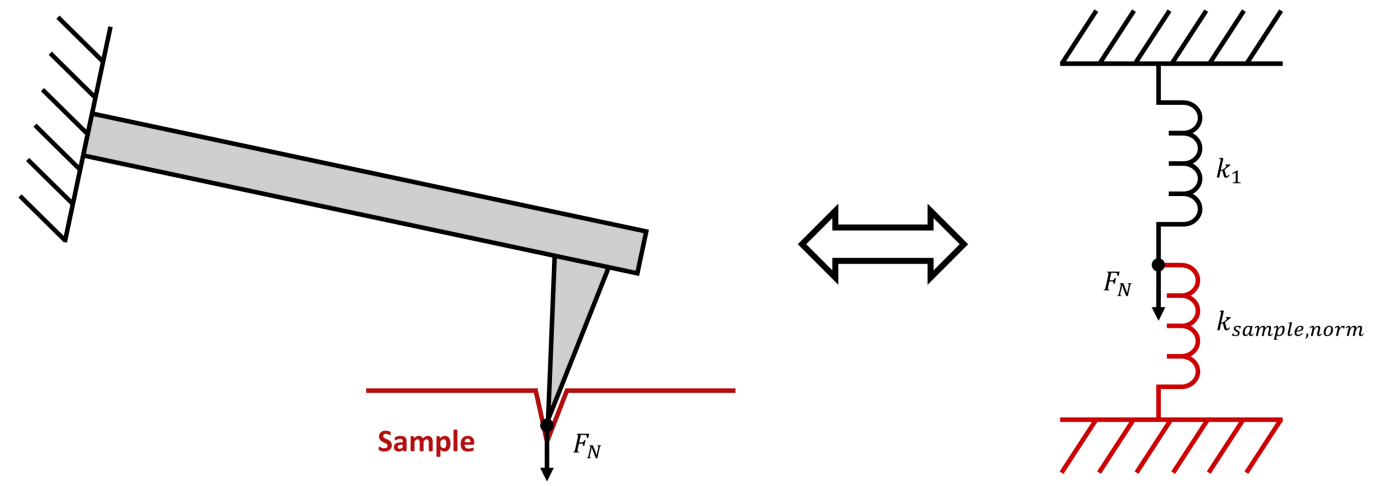

a
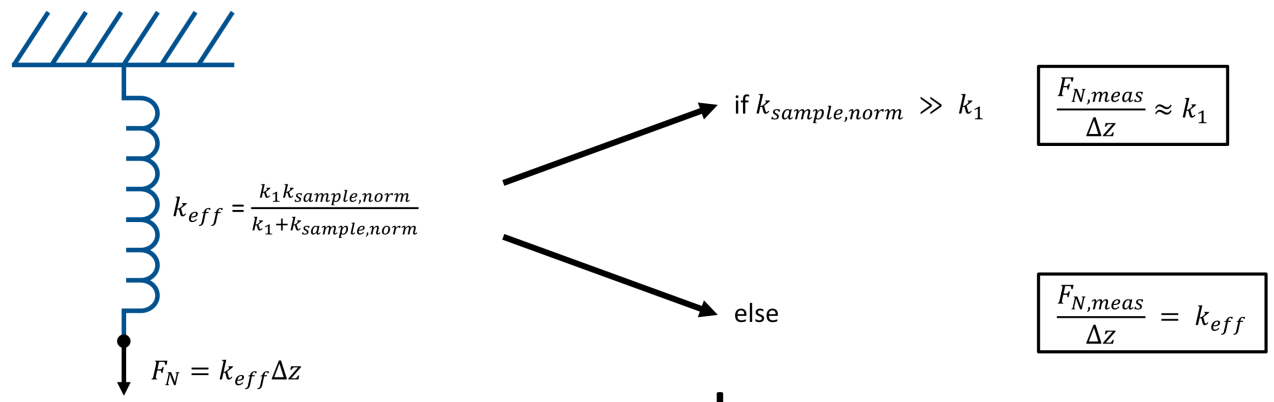

b

Figure 4: (a) Cantilever in contact with the sample surface modeled by two springs in series, $k_{1}$ and $k_{\text {sample,norm, }}$, representing the cantilever spring constant and the normal sample stiffness, respectively. The arrow $F_{N}$ represents the applied normal force. (b) Effective spring constant $k_{\text {eff }}$ and values of the ratio between measured applied normal force $F_{N \text {,meas }}$ and $Z$-displacement $\Delta z$ as a function of the sample stiffness. 
the value of $k_{\text {eff }}$ approaches $k_{1}$, so that beyond a certain value of

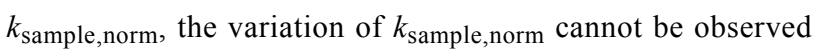
anymore in the $F_{N}$, meas $(Z)$ curve. This effect is evidenced in Figure 2 and Figure 3 (right) with the $F_{N}$, meas $(Z)$ curve (black dots), where the inverse of the slope of the linear fit (red line) shows a value close to the cantilever spring constant as given in Table 1.

\begin{tabular}{|c|c|}
\hline sample & inverse of slope $\left(\mathrm{N} \cdot \mathrm{m}^{-1}\right)$ \\
\hline LLDPE & 0.315 \\
\hline PP & 0.308 \\
\hline PS & 0.313 \\
\hline FDTS & 0.324 \\
\hline PTFE & 0.320 \\
\hline
\end{tabular}

\section{Sample surface deformations and evolution of stress during indentation}

The existence of a plastic and an elastic deformation phase is explained by the evolution of stress $\sigma$ (ratio between the applied normal force $F_{N}$ and the cross section $A$ of the cantilever tip) during indentation (Figure 5). At the beginning of the indenta- tion, after setting the tip on the sample surface, stress is increased from a normal force with an initial value $0 \mathrm{nN}$. The evolution of stress can be split into three phases. In the first phase, if the applied normal force increases gently or if the contact surface between the tip apex and the surface is large enough, the generated stress increases gently enough to deform the sample surface elastically. This elastic phase does not occur if the steps of the $Z$-scanner, which are responsible for increasing the applied normal force, are too big. In this case, the generated stress is strong enough to deform the surface plastically, and phase 2 is initiated. In the second phase, due to the assumed pyramidal geometry of the tip, the contact area is small enough and the applied normal force is strong enough to create a stress that deforms the sample surface plastically. In the third phase, the tip geometry and the increase of the indentation depth result in a larger increase in the contact area than that produced by the applied normal force, thus decreasing the stress on the sample surface. When the stress decreases below a threshold value, $\sigma_{0}$, the sample starts deforming elastically.

\section{Linear relation between $\Delta f_{1}$ and $\Delta f_{2}^{2}$ and determination of $\alpha\left(A_{1}\right)$}

The linearity relation between $\Delta f_{1}$ and $\Delta f_{2}^{2}$ is shown in Figure 2 and Figure 3 (curves on the right) where the quasi-invariance of the ratio $\Delta f_{2}^{2} / \Delta f_{1}$ in the elastic deformation phase and in the
Phase 1

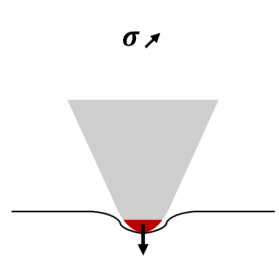

$F_{N} \rtimes$

$A \pi$
Phase 2

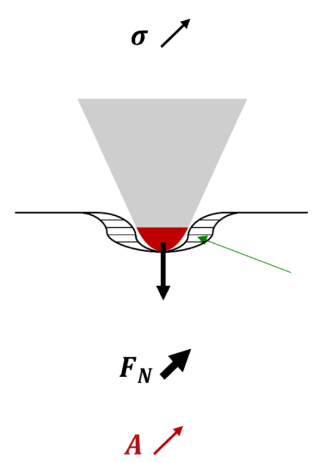

Plastic deformation
Phase 3
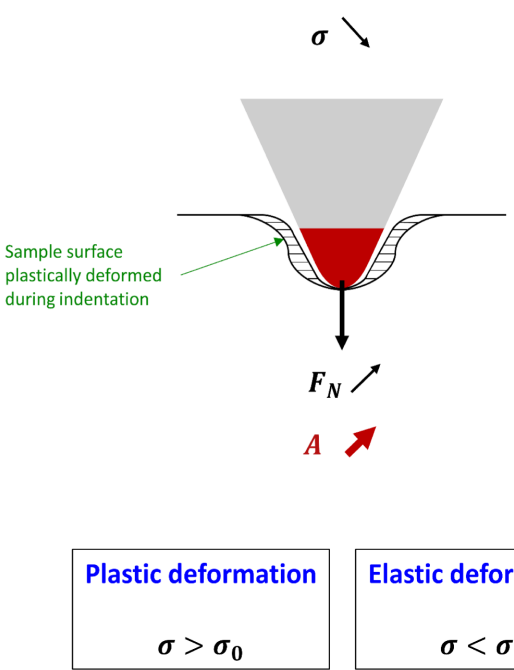

\begin{tabular}{|c|}
\hline Elastic deformation \\
$\sigma<\sigma_{0}$ \\
\hline
\end{tabular}

Figure 5: Three phases characterizing the evolution of stress $\sigma$ during indentation. This evolution is responsible for the plastic and elastic deformation of the sample. In phase 1 , in the presence of a sufficiently weak applied normal force $F_{N}$ or a sufficiently large cross section $A$, the generated stress is small enough to deform the surface elastically. This initial elastic phase can only be observed, if the steps used for the Z-scanner are small enough. If the steps are too big, the evolution of $\sigma$ is directly initiated with phase 2 . In phase 2, the large value of $F_{N}$ and its fast increase compared to A generate a stress large enough to deform the surface plastically. In phase 3 , the geometry of the tip results in a fast increase of the contact area, and hence of $A$, compared to the moderate increase in $F_{N}$, which decreases the stress. When the stress decreases below a threshold value $\sigma_{0}$, the deformation becomes elastic. The red area of the tip apex describes the contact area of the tip. The striped area shows the region of the sample plastically deformed during indentation. The arrow at the tip apex represents the applied normal force $F_{N}$; the arrows on the right of $\sigma, F_{N}$ and $A$ characterize the increase speed (bold for fast) and magnitude (length). 
upper part of the plastic deformation phase can be observed. The mean value of the linear coefficient for each sample and the corresponding maximum deviation in percent are reported in Table 2. The weak deviation in the upper part of the plastic de-

$\begin{aligned} & \text { Table 2: Mean value of } \Delta f_{2}^{2} / \Delta f_{1} \text { and corresponding maximum devia- } \\
& \text { tion (in percent) in the elastic deformation phase and the upper region } \\
& \text { of the plastic deformation phase. }\end{aligned}$
\begin{tabular}{llll} 
sample & $\begin{array}{l}\text { mean } \\
\Delta f_{2}^{2} / \Delta f_{1}(\mathrm{~Hz})\end{array}$ & $\begin{array}{l}\text { max. dev. elastic } \\
\text { phase }(\%)\end{array}$ & $\begin{array}{l}\text { max. dev. plastic } \\
\text { phase (\%) }\end{array}$ \\
\hline LLDPE & 48,720 & 0.89 & 3 \\
PP & 239,738 & 1.77 & 5 \\
PS & 417,224 & 1.38 & 6.7 \\
FDTS & 61,614 & 0.1 & 0.8 \\
PTFE & 67,981 & 0.52 & 1
\end{tabular}

formation phase suggests that setpoints for $F_{N}$ in that region can be used to investigate the sample elasticity. The parameter $\alpha\left(A_{1}\right)$ was determined by dividing the nominal value of the Young's modulus of PTFE by the mean value of $\Delta f_{2}^{2} / \Delta f_{1}$ of PTFE. The computation yielded a value of $7,355 \mathrm{~Pa} \cdot \mathrm{Hz}^{-1}$.

\section{Investigation of sample elastic modulus}

The elastic modulus of the sample was then investigated with setpoints of the main feedback in the highest region of the plastic phase of $44.4 \mathrm{nN}$ for LLDPE, $29.6 \mathrm{nN}$ for PP, $29.6 \mathrm{nN}$ for PS and $37 \mathrm{nN}$ for FDTS. The results of the scannings are shown in Figure 6 and Figure 7 for topography (panel a) and elastic modulus (panel b). The histograms in Figure $6 \mathrm{c}$ and Figure $7 \mathrm{c}$ correspond to the distribution of the elastic modulus for each map in Figure $6 \mathrm{~b}$ and Figure $7 \mathrm{~b}$. Because polymers are viscoelastic materials, the elastic modulus $E_{\text {eff,meas }}$ measured on

\section{LLDPE}
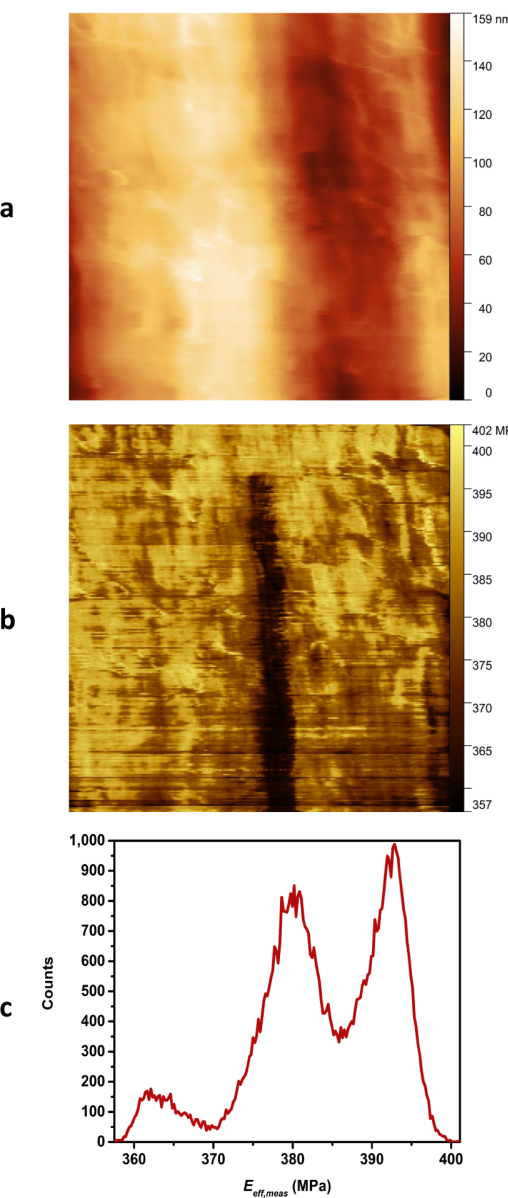

PP
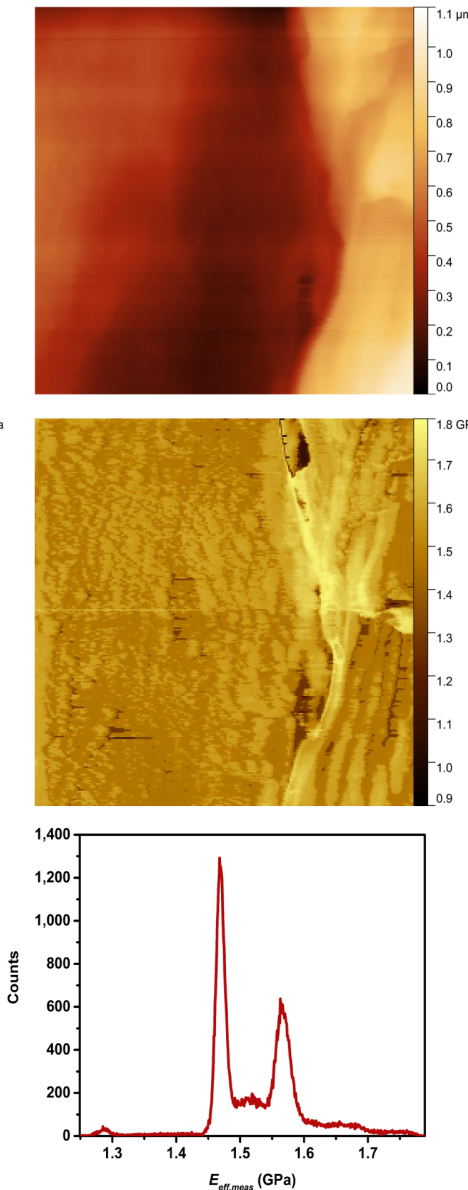

PS
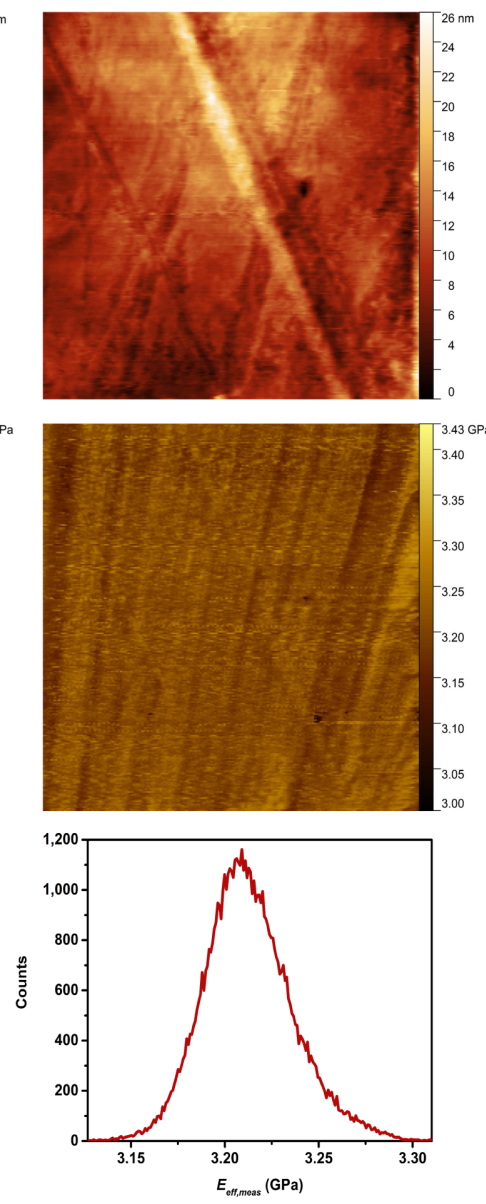

Figure 6: Mapping of (a) topography and (b) elastic modulus of $2.5 \mu \mathrm{m} \times 2.5 \mu \mathrm{m}$ areas of the LLDPE, PP and PS samples. The measurements were performed in contact by applying a constant normal force $F_{N}$ of $44.4 \mathrm{nN}$ for LLDPE and $29.6 \mathrm{nN}$ for PP and PS, and by exciting the two first flexural modes of the cantilever with an amplitude of $A_{1}=22 \mathrm{mV}$ and $A_{2}=5 \mathrm{mV}$, respectively, for the first and second mode. The histograms in panel (c) show the distribution of elastic modulus $E_{\text {eff,meas }}$ in the maps in panel (b). 


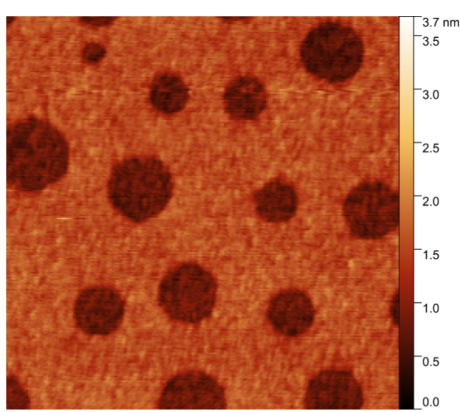

a
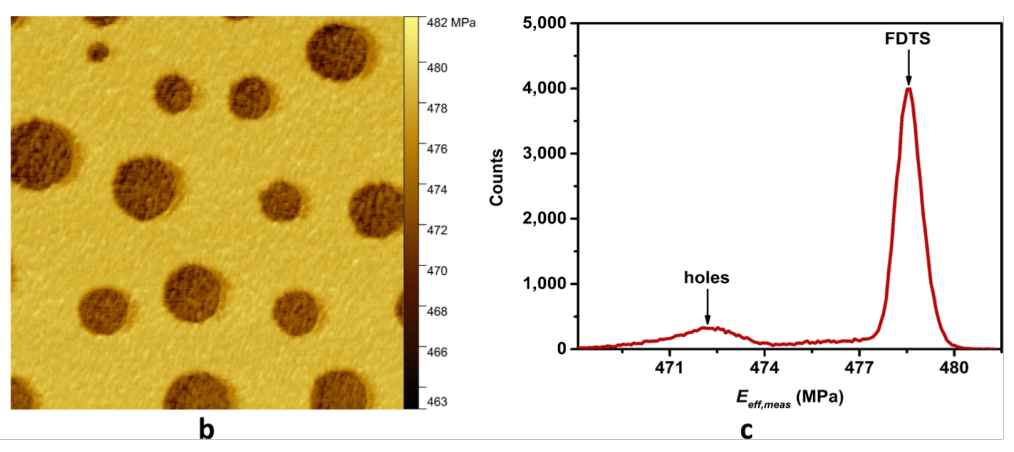

Figure 7: Mapping of (a) topography and (b) elastic modulus of a $2.5 \mu \mathrm{m} \times 2.5 \mu \mathrm{m}$ area of the FDTS + SiOx SAM sample. The measurements were performed in contact by applying a constant normal force $F_{N}$ of $37 \mathrm{nN}$, and by exciting the two first flexural modes of the cantilever with an amplitude of $A_{1}=22 \mathrm{mV}$ and $A_{2}=5 \mathrm{mV}$ respectively for the first and second mode. The histogram in panel (c) shows the distribution of elastic modulus $E_{\text {eff,meas }}$ in panel (b).

the investigated samples in dynamic mode corresponds to the storage modulus. As the measured contact resonances are quite large compared to the inverse of typical material relaxation time, we can assume that the measured storage modulus is independent on the frequency. The measurements yielded values in the range of the Young's moduli of bulk LLDPE, PP and PS as seen in Table 3. The investigation also evidenced regions of different elastic moduli, as seen in the histograms of LLDPE, PP and the SAM: LLDPE shows three different peaks centered at 362, 380 and $393 \mathrm{MPa}, \mathrm{PP}$ shows two peaks centered at 1.468 $\mathrm{GPa}$ and $1.565 \mathrm{GPa}$ and the SAM shows two peaks centered at 472 and $478.5 \mathrm{MPa}$. Finally, the value of the storage modulus of
FDTS gives an estimate of the Young's modulus of the FDTS monolayer.

\section{Effect of tip functionalization on the SiOx elasticity peak of the SAM sample}

The proximity of the peaks of FDTS and SiOx holes can be explained by the fact that during scanning, some material of FDTS has gathered around the tip, so that the relatively soft FDTScoating of the tip is sensed (see also Figure 8 ). The effect of collecting Teflon-like molecules with AFM tips has been known for a long time. It has been successfully used for the topographic imaging of the $\mathrm{Si}(111)$ surface with atomic resolu-

Table 3: Measured storage moduli $E_{\text {eff,meas }}$ and literature Young's modulus values of bulk materials $E_{\text {sample,lit }}$ of LLDPE, PP, PS and FDTS. The values of $E_{\text {eff,meas }}$ are in the range of the Young's moduli of the bulk materials. No literature value is available for FDTS.

\begin{tabular}{lll} 
sample & $E_{\text {eff,meas }}(\mathrm{GPa})$ & $E_{\text {sample,lit }}(\mathrm{GPa})$ \\
\hline LLDPE & $0.362 \pm 0.004,0.38 \pm 0.004,0.393 \pm 0.005$ & $0.3-0.7[25]$ \\
PP & $1.468 \pm 0.009,1.565 \pm 0.015$ & $0.896-1.55[26]$ \\
PS & $3.21 \pm 0.02$ & $2.28-3.34[26]$ \\
FDTS & $0.4785 \pm 0.0005$ &
\end{tabular}
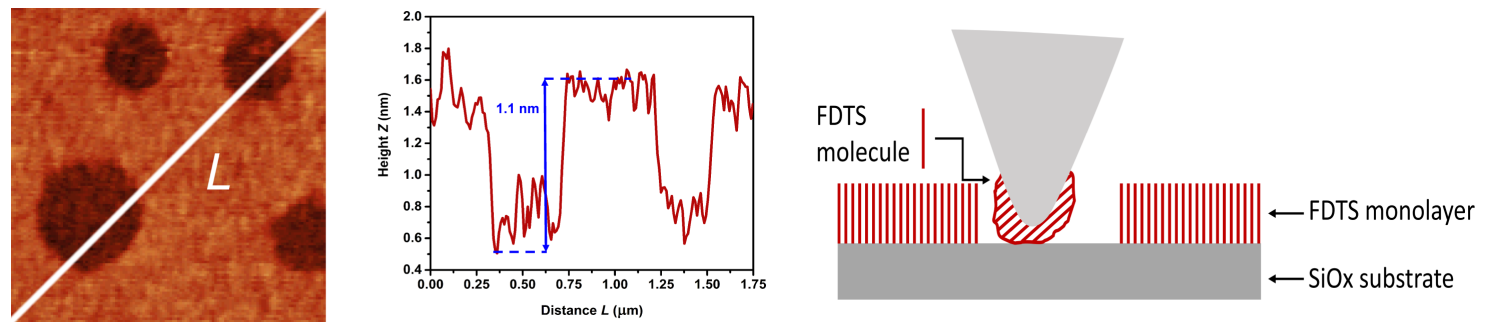

Figure 8: Line profile of the SiOx holes in the topographical mapping of the SAM showing their relative height and width due to the functionalization of the cantilever tip with FDTS material. On the right, a schematic of the tip-sample interaction is depicted. Loosely bound FDTS molecules are able to migrate to the AFM tip and can therefore functionalize by forming a thin layer on the tip apex. 
tion. Howald et al. [27] studied the $\operatorname{Si}(111) 7 \times 7$ reconstruction in UHV by contact force microscopy. They observed adhesive forces of up to $103 \mathrm{nN}$ between the Si tip and the Si(111) surface. By coating the tip with PTFE (Teflon), they could reduce the sticking forces to $10 \mathrm{nN}$. A short scan on a PTFE sample led to a reliable coating of the tip for the subsequent AFM scan on silicon. Since our sample consists of a perforated Teflon-like monolayer, the cantilever tip is most likely constantly functionalized with a thin layer of FDTS. This leads to the observed soft elasticity in- and outside the holes. The stability of this functionalization during the scan can be verified with topographic images. The holes show a depth slightly below $1.2 \mathrm{~nm}$, which is the height of the FDTS monolayer, as seen in Figure 8. If the tip coating was worn-off during the scan, larger or at least partially deeper holes would appear.

\section{Conclusion}

We present a robust method for the quantitative determination of local elastic modulus of sample under ambient conditions (humidity and temperature). It combines the simplicity of the theoretical model developed by Herruzo et al. [7] for the determination of sample elasticity from $1 \mathrm{MPa}$ to $3 \mathrm{GPa}$ with a robust measuring method based on contact resonances. The measurements consist of tracking the first and second flexural contact resonances of the cantilever to determine the frequency shifts $\Delta f_{1}$ and $\Delta f_{2}$ relative to the corresponding resonances in free space in order to compute the elastic modulus.

The linear relation between $\Delta f_{1}$ and $\Delta f_{2}^{2}$ established in [7] can be determined from the measurement of $\Delta f_{1}$ and $\Delta f_{2}$ as functions of the $Z$-displacement of the piezoelectric scanner. These curves were measured on four polymers, i.e., LLDPE, PP, PS, PTFE, and a Teflon-like fluorinated SAM.

Analysis of these curves evidenced the existence of plastic and elastic deformation phases. The two subsequent phases are explained by the evolution of stress $\sigma$ during indentation by the cantilever tip. Plastic deformation begins when sufficient stress is applied to the surface. The slow increase in tip cross section $A$ compared to the applied normal force $F_{N}$ results in an increase in $\sigma$ and leads to the plastic deformation of the sample surface. The geometrical shape of the tip, assumed to be pyramidal, results in a fast increase in tip cross section compared to the increase in applied normal force $F_{N}$ when the tip goes deeper into the surface. This, in turn, decreases the stress. When the $\sigma$ is below a threshold value, the surface begins to deform elastically.

The elastic phase is characterized by a linear relation between the frequency shifts $\Delta f_{1}$ and $\Delta f_{2}$ and the $Z$-displacement of the piezoelectric scanner. A linear relation between the measured normal force $F_{N \text {,meas }}$ and the displacement in both phases can also be observed if the spring constant of the cantilever is small compared to the normal sample stiffness. This linearity does not reflect the variation in normal sample stiffness but the excessive compliance of the cantilever. The use of cantilevers with higher spring constants is actually limited for technical reasons. Indeed, for cantilevers with a too high spring constant, the second flexural contact resonance is out of the bandpass of the vertical deflection channel in most of the microscope heads.

The analysis of the $\Delta f_{2}^{2} / \Delta f_{1}\left(F_{N \text {,meas }}\right)$ curve showed that setpoints for the applied normal force in the highest region of the plastic deformation phase could be used without causing a large deviation in the elasticity compared with the results we would obtain in the elastic deformation phase. The elasticity of LLDPE, PP, PS, and the SAM was then investigated. As polymers are viscoelastic materials, the elastic modulus $E_{\text {eff,meas }}$ measured in dynamic mode corresponds to the storage modulus. We assumed a frequency independence of the measured storage modulus as the measured contact resonances are quite large compared to the inverse of typical material relaxation times. The measurements showed values for the storage modulus in the range of the Young's moduli for bulk materials for LLDPE, PP and PS.

The method can also distinguish variations in the elasticity of surface such as LLDPE, PP and the FDTS SAM. The investigated SAM consists of a monolayer of FDTS patterned with circular $1.2 \mathrm{~nm}$ deep holes. Due to the functionalization of the cantilever tip by SAM molecules, a similar stiffness is measured inside and outside the holes. Finally, the measured value of the storage modulus of $478.5 \pm 0.5 \mathrm{MPa}$, which is close to the value for bulk PTFE (500 MPa), can be used as an estimation of the Young's modulus of the FDTS monolayer.

\section{Supporting Information}

\section{Supporting Information File 1}

Supporting information features the analytical expression for normal sample stiffness based on Hurley and Turner's equations.

[http://www.beilstein-journals.org/bjnano/content/ supplementary/2190-4286-9-1-S1.pdf]

\section{Acknowledgements}

We would like to thank the Swiss National Science Foundation (SNF), the Swiss Nanoscience Institute (SNI) and the Commission for Technology and Innovation (CTI) for financing the project. Thomas Schimmel and Stefan Walheim acknowledge the financial support by the Baden-Württemberg Stiftung within 
the Research Network "Functional Nanostructures". We especially thank Alexis Baratoff for rewarding discussions about contact AFM and polymer elasticity.

\section{References}

1. Kasas, S.; Dietler, G. Probing the Nanomechanical Properties of Viruses, Cells and Cellular Structures. In Life at the Nanoscale: Atomic Force Microscopy of Live Cells; Dufrêne, Y., Ed.; Pan Stanford Publishing: Singapore, 2011; pp 335-352.

2. Dai, S.; Zhao, J.; He, M.-r.; Wang, X.; Wan, J.; Shan, Z.; Zhu, J. Nano Lett. 2015, 15, 8-15. doi:10.1021/nl501986d

3. Lu, H.; Meng, X. Sci. Rep. 2015, 5, 16939. doi:10.1038/srep16939

4. Binnig, G.; Quate, C. F.; Gerber, C. Phys. Rev. Lett. 1986, 56, 930-934. doi:10.1103/PhysRevLett.56.930

5. Tamayo, J.; Garcia, R. Appl. Phys. Lett. 1997, 71, 2394-2396. doi:10.1063/1.120039

6. Hurley, D. C.; Turner, J. A. J. Appl. Phys. 2007, 102, 033509. doi:10.1063/1.2767387

7. Herruzo, E. T.; Perrino, A. P.; Garcia, R. Nat. Commun. 2014, 5, 3126. doi:10.1038/ncomms4126

8. Bubendorf, A. Elasticity of Polymers Investigated by Atomic Force Microscopy. Ph.D. Thesis, University of Basel, Basel, Switzerland, 2015.

9. Rabe, U. Atomic Force Acoustic Microscopy. In Applied Scanning Probe Methods II; Bhushan, B.; Fuchs, H., Eds.; Springer: Berlin, Germany, 2006; pp 37-90.

10. Rabe, U.; Turner, J.; Arnold, W. Appl. Phys. A 1998, 66, S277-S282. doi:10.1007/s003390051145

11. Rabe, U.; Arnold, W. Appl. Phys. Lett. 1994, 64, 1493-1495. doi:10.1063/1.111869

12. Rabe, U.; Amelio, S.; Kester, E.; Scherer, V.; Hirsekorn, S.; Arnold, W. Quantitative determination of contact stiffness using atomic force acoustic microscopy. Ultrasonics; Elsevier: Amsterdam, Netherlands, 2000; Vol. 48, pp 430-437.

13. Rabe, U.; Amelio, S.; Kopycinska, M.; Hirsekorn, S.; Kempf, M.; Göken, M.; Arnold, W. Surf. Interface Anal. 2002, 33, 65-70. doi:10.1002/sia.1163

14. Rabe, U.; Kopycinska-Müller, M.; Hirsekorn, S. Atomic Force Acoustic Microscopy. In Acoustic Scanning Probe Microscopy; Marinello, F.; Passeri, D.; Savio, E., Eds.; Springer: Berlin, Germany, 2012; pp 123-153.

15. Lozano, J. R.; Garcia, R. Phys. Rev. Lett. 2008, 100, 076102. doi:10.1103/PhysRevLett.100.076102

16. Garcia, R.; Herruzo, E. T. Nat. Nanotechnol. 2012, 7, 217-226. doi:10.1038/nnano.2012.38

17. Rodriguez, T. R.; Garcia, R. Appl. Phys. Lett. 2004, 84, 449-451. doi:10.1063/1.1642273

18. Proksch, R. Appl. Phys. Lett. 2006, 89, 113121. doi:10.1063/1.2345593

19. Martínez, N. F.; Lozano, J. R.; Herruzo, E. T.; Garcia, F.; Richter, C.; Sulzbach, T.; Garcia, R. Appl. Phys. Lett. 2008, 19, 384011. doi:10.1088/0957-4484/19/38/384011

20. Herruzo, E. T.; Garcia, R. Beilstein J. Nanotechnol. 2012, 3, 198-206. doi:10.3762/bjnano.3.22

21. Garcia, R.; Proksch, R. Eur. Polym. J. 2013, 49, 1897-1906. doi:10.1016/j.eurpolymj.2013.03.037

22. Meyer, E.; Gyalog, T.; Overney, R. M.; Dransfeld, K., Eds. Friction and Rheology on the Nanometer Scale; World Scientific Publishing Co Pte Ltd: Singapore, 1998.
23. Rast, S.; Wattinger, C.; Gysin, U.; Meyer, E. Rev. Sci. Instrum. 2000 , 71, 2772-2775. doi:10.1063/1.1150690

24. Huang, C.; Moosmann, M.; Jin, J.; Heiler, T.; Walheim, S.; Schimmel, T. Beilstein J. Nanotechnol. 2012, 3, 620-628. doi:10.3762/bjnano.3.71

25. Lechner, M. D. Polymers. In Springer Handbook of Condensed Matter and Materials Data; Martienssen, W.; Warlimont, H., Eds.; Springer: Berlin, Germany, 2005; pp 477-552.

26. Materials Data Book. Cambridge University Engineering Department: Cambridge, United Kingdom, 2003;

http://www-mdp.eng.cam.ac.uk/web/library/enginfo/cueddatabooks/mat erials.pdf (accessed June 21, 2017).

27. Howald, L.; Lüthi, R.; Meyer, E.; Güntherodt, H.-J. J. Phys.: Condens. Matter 1994, 93, 267-268. doi:10.1007/BF01312696

\section{License and Terms}

This is an Open Access article under the terms of the Creative Commons Attribution License (http://creativecommons.org/licenses/by/4.0), which permits unrestricted use, distribution, and reproduction in any medium, provided the original work is properly cited.

The license is subject to the Beilstein Journal of Nanotechnology terms and conditions: (http://www.beilstein-journals.org/bjnano)

The definitive version of this article is the electronic one which can be found at: doi:10.3762/bjnano.9.1 